\title{
The Effect of Portulaca Oleracea Seeds on Anthropometric Measurements in Asthmatic Patients with Overweight and Obesity
}

\author{
Seyyedeh Zahra Hosseini ${ }^{1}$, Beitollah Alipour ${ }^{2}$, khalil Ansarin $^{3}$, Akbar Sharifi $^{4}$ \\ ${ }^{1}$ Faculty of Nutrition, Tabriz University of Medical Sciences, Tabriz, Iran \\ ${ }^{2}$ Department of Community Nutrition, Faculty Nutrition, Tabriz University of Medical Sciences, Tabriz, Iran \\ ${ }^{3}$ Pulmonary Critical Care Medicine, Tuberculosis and Lung Disease Research Center, Tabriz, Iran \\ ${ }^{4}$ Internal Medicine, Tabriz University of Medical Sciences, Tabriz, Iran
}

Email address:

alipourb@tbzmed.ac.ir (B. Alipour)

\section{To cite this article:}

Seyyedeh Zahra Hosseini, Beitollah Alipour, khalil Ansarin, Akbar Sharifi. The Effect of Portulaca Oleracea Seeds on Anthropometric Measurements in Asthmatic Patients with Overweight and Obesity. Journal of Food and Nutrition Sciences.

Vol. 3, No. 3, 2015, pp. 103-107. doi: 10.11648/j.jfns.20150303.14

\begin{abstract}
Background and Objective: Many studies show that there is a relationship between asthma and obesity. Weight management is important in patients with asthma. In some countries, people used Portulaca oleracea seeds for lose weight. The experiment is designed to study the effect of Portulaca oleracea seeds on anthropometric measurements in asthmatic patients with overweight and obesity. Material and method: In a randomized clinical trial, 37 patients with asthma disease that refer to clinics of Tabriz University of medical sciences divided to intervention and control groups. First group ( $\mathrm{n}=18$ ) received protocol treatment and 10g/day Portulaca oleracea seeds in two doses with two glasses of water, while participants in second group $(n=19)$ had only their protocol treatment and drank two glasses of water in every day for 8 weeks. At the baseline and end of study, anthropometric measurements were obtained for each person and dietary intake and physical activity questionnaires were completed. Results: Mean age and history of asthma for participants at baseline was $45.13 \pm 9.2$ and $9.9 \pm 8.1$ years, respectively. Comparing of anthropometric measurements including weight, body mass index (BMI), waist circumference (WC) and hip circumference (HC), waist-to-hip ratio (WHR) between two groups indicated any significant differences between two groups after consumption of seeds $(p>0.05)$. Conclusion: Administration of Portulaca oleracea seeds could not decrease anthropometric measurements in asthmatic patients with overweight and obesity.
\end{abstract}

Keywords: Asthma, Portulaca Oleracea Seed, Obesity, Overweight

\section{Introduction}

The prevalence of asthma and obesity has increased in recent decades (1) So that nearly 300 million people of all ages and races are affected by asthma worldwide (2, 3). Also, it is anticipated that approximately 2.3 billion adults are overweight and more than 700 million adults are obese patients by 2015 (1).

Based on epidemiological studies, there is a significant relationship between BMI with control and severity of asthma (4). Obesity is considered as an important factor in this relationship because it can antedate the risk of asthma and create a new phenotype of the disease with less control, increased symptoms and exacerbations (5). Systemic inflammation and oxidative stress due to obesity influence on airway inflammation and cause to change eosinophilic inflammation (6). Therefore, attention to weight management is obviously essential for better control and improving the effectiveness of drugs.

Portulaca Oleracea is an annual weed (7). This plant has different names in various cultures such as "Rudravant" in India, "Berbin" in Iraq, "Purslane" in United States and "Perpin" in Iran $(8,9)$. Portulaca Oleracea is widely used in the world so far as it is known as the eighth herb (10) and called "universal panacea" too (11). Chemical compounds in different parts of the plant contain carbohydrates, protein, calcium, zinc, potassium, sodium $(12,13)$, manganese, iron, phosphorus (14), selenium and vitamins A, E, C, fiber, coenzyme $\mathrm{Q}_{10}(15)$, carotene, essential fatty acids, vitamins (16).

Pharmacological effects of Portulaca Oleracea like 
hypoglycemic (17), hypocholesterolemic (18), anti-diabetes (19) have demonstrated in several studies. In some country, seeds of Portulaca Oleracea are used to lose weight (20) and availability to seeds was easier than green parts of this plant. Respectively, we decided to design a study to examine the effect of P.oleracea seeds on weight lost and anthropometric measurements in asthmatic patients with overweight and obesity .

\section{Material and Method}

Methods are presented as blow:

\subsection{Preparation of Portulaca Oleracea Seeds}

The dried P.oleracea seeds were purchased from the reliable herbal medicine market in Tabriz, East Azarbaijan Province, Iran. This store was approved by the Tabriz University of Medical Sciences and an evidence specimen of seeds was deposited in the departmental nutrition for future reference. After checking of seeds, $10 \mathrm{~g}$ of them was dumped to container and 56 dishes put in the package for every participant.

\subsection{Participants}

In a clinical trial study, forty asthmatic patients who refer to subspecialty clinics of Tabriz University of Medical Sciences from Jun 2012 to May 2013 were recruited. First two Pulmonologist confirmed asthma disease for every one according to the Global Strategy for Asthma (GINA) and then inclusion criteria including 18-80 years old, body mass index $(\mathrm{BMI})>25$ and history of asthma duration at least 1 year were check for each person. If they were smoking, having other lung disorders and respiratory tract infections, pregnant or lactating, kidney, liver, or cardiovascular diseases, taking any multivitamin and mineral and dietary supplements containing antioxidants and omega- 3 in 2 months ago, any change in drug type and dosage, having a history of oxalate stones, having a exacerbation in last month that led to hospitalization, follow a diet, taking contraceptive pills, they excluded from the study. At the first of study, a written informed consent was obtained from each participant.

\subsection{Trial Design}

Participants were randomly divided to intervention $(n=20)$ and control $(\mathrm{n}=20)$ groups. In the interventional group, they received treatment protocol and daily took $10 \mathrm{~g}$ P.oleracea seeds (one container) in two doses (5gm) in morning (fasting) and before dinner with a glass of water at every turn for 8 weeks. In control group, patients received their treatment protocol and drank two glasses of water at the same times of first group in a day for 8 weeks. There was no placebo for this study. The treatment protocol was consisted of $\beta_{2^{-}}$ adrenergic agonists with or without corticosteroids.

In before and after of study, weight and height of them were measured with the nearest $0.1 \mathrm{~kg}$ and $0.1 \mathrm{~cm}$, respectively by a research assistant while patients wore light clothing and were barefoot. Body mass index was calculated as weight in kilograms divided by the square of the height in meters. Measuring waist circumference (halfway between the 10th rib laterally and the most superior part of the anterior superior iliac crest) and hip circumference (largest circumference between the waist and knees) using a tape with a nearest $0.1 \mathrm{~cm}$ was carried out and then waist-to-hip ratio was calculated.

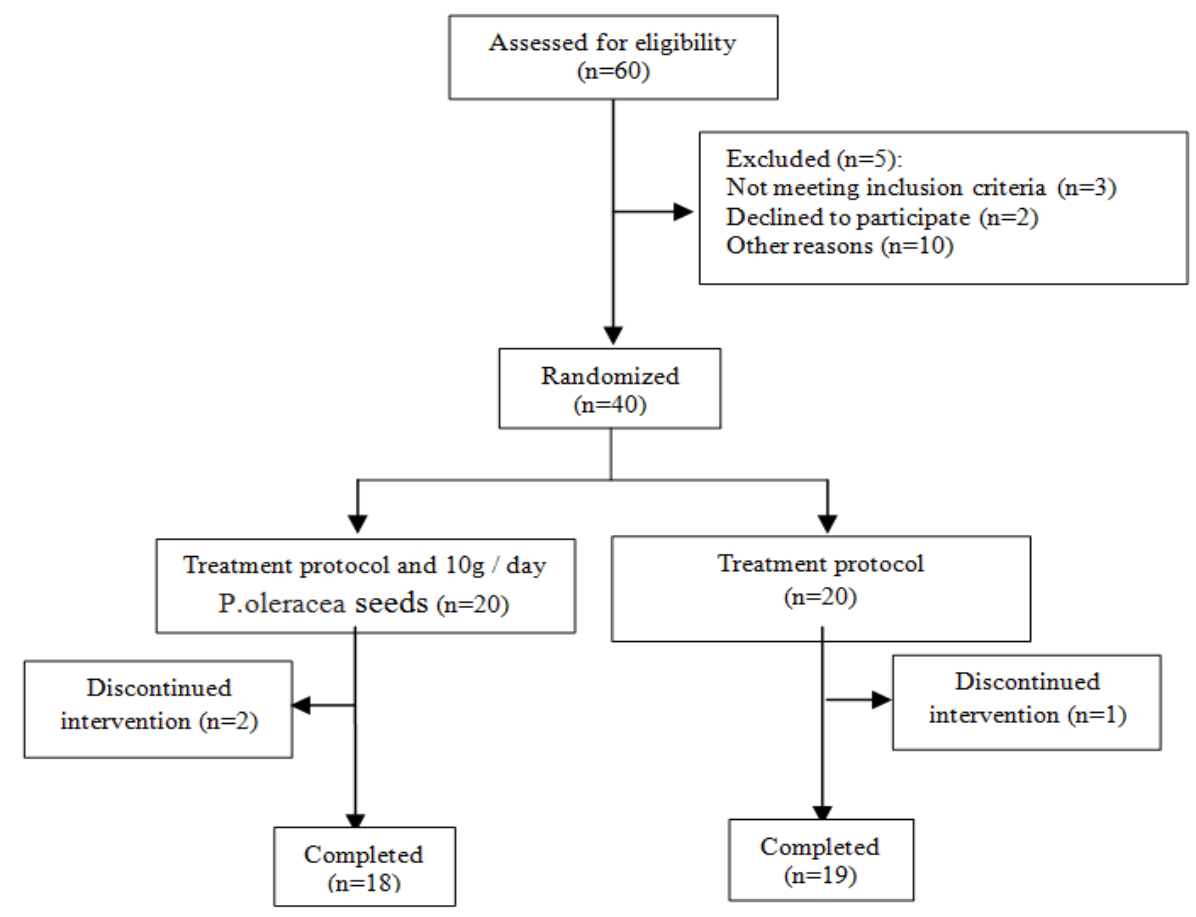

Fig. 1. Study design 
For assessment of dietary intake and physical activity, 24hour dietary recall questionnaire for 3-days and Metabolic Equivalents (MET) questionnaire for one-day were completed by a student of nutrition for each person at the beginning and end of trial. Dietary data were analyzed using the Nutritionist 4 software (First Databank Inc., Hearst Corp., San Bruno, CA, USA).

According to the parameter of BMI in the study of ElSayed (20) et al and a two-sided 95\% significant level and a power of $80 \%$, sample size was determined 20 patients per group with dropout rate of $25 \%$. Finally, sample size was calculated.

\subsection{Statistical Analysis}

Data were analyzed using the SPSS software version 16.0 (SPSS Inc., Chicago, IL, USA). The results were expressed as mean (standard deviation). Chi-square analysis was used for quality measures. The normality of the distribution of data was determined by Kolmogorov-Smirnov test. Comparison of data inside and between two groups was made using paired t-test and Independent t-test respectively. Mann-Whitney and Wilcoxon test was used for non-normally distributed data. Analysis of covariance (ANCOVA) was used to identify any differences between the two groups after intervention, and was adjusted for the baseline measurements and covariates. Values of $\mathrm{P}<0.05$ were considered statistically significant.

\section{Results}

After 8 weeks, thirty seven participants (18 intervention group and 19 control group) were completed this study (fig 1). The mean of age and median of asthma duration were 44 \pm 8.8 and $10 \pm 7.5$ years in intervention group and $46 \pm 9.6$ and $9.6 \pm 8.8$ years in control group, respectively.

As results of this study, there were no significant differences about age, duration of asthma and height of patients in both groups at the baseline $(P>0.05)$. The results of comparison of demographic information such as sex, education, job, habitat, family history of asthma and exposure to cigarette are shown in Table1. According to it, baseline characteristics of patients were all similar in both groups $(P>0.05)$.

Table 1. Demographic characteristics of the study participants ( $n=37)$.

\begin{tabular}{llll}
\hline characteristics & $\begin{array}{l}\text { P.oleracea seeds group }(\mathrm{n}=18) \\
\text { Frequency }(\%)\end{array}$ & $\begin{array}{l}\text { Control group }(\mathrm{n}=19) \\
\text { Frequency (\%) }\end{array}$ & 0.17 \\
\hline $\begin{array}{l}\text { Sex (female) } \\
\text { Education }\end{array}$ & $12(63)$ & $15(83)$ & 0.19 \\
Illiterate & $5(28)$ & $4(33)$ & 0.14 \\
Literacy & $13(88)$ & $15(77)$ & \\
Habitat & & & \\
City & $16(89)$ & $16(84)$ & 0.6 \\
Village & $2(11)$ & $3(16)$ & \\
Job & $14(78)$ & $10(53)$ & \\
Housekeeper & $1(5.5)$ & $2(10.5)$ & 0.9 \\
Employer & 0 & $2(10.5)$ & \\
Farmer/worker & $3(16.5)$ & $5(26)$ & \\
Other & $7(51.6)$ & $7(37)$ & \\
Family history of asthma & $11(48.4)$ & $12(63)$ & \\
Yes & $7(51.6)$ & $9(47)$ & \\
No & $11(48.4)$ & $10(53)$ & \\
Exposure to cigarette (inactive) & & & \\
Yes & & & \\
No & & & \\
\hline
\end{tabular}

*Chi-square

As shown by Table2, comparing of dietary intake and physical activity showed no significant differences at

beginning and after 8 weeks between two groups $(P>0.05)$.

Table 2. Dietary intake and physical activity of participants at the baseline and end of the study.

\begin{tabular}{|c|c|c|c|c|c|}
\hline \multirow{2}{*}{ Parameter } & \multicolumn{2}{|c|}{ P.oleracea seeds group $(n=18)$} & \multicolumn{2}{|l|}{ Control group $(n=19)$} & \multirow{2}{*}{$\operatorname{MD}(95 \% C I)^{a}$} \\
\hline & Baseline(mean(SD)) & 8 weeks(mean(SD)) & Baseline(mean(SD)) & 8 weeks (mean(SD)) & \\
\hline Energy(kcal) & $1732(405)$ & $1804(624)$ & $1856(392)$ & $1814(421)$ & $-9.5(-362.8$ to 343.9$)$ \\
\hline Carbohydrate(g) & $243(62)$ & $244(79.5)$ & $274(79)$ & $256(74.56)$ & $-11.99(--63.42$ to39.4) \\
\hline $\operatorname{Protein}(\mathrm{g})$ & $59(21)$ & $60(21.5)$ & $65(19)$ & $62(21)$ & $-1.5(-15.7$ to 14.65$)$ \\
\hline Total fat(g) & $59.5(16)$ & $66(31)$ & $57.5(15)$ & $60.5(14)$ & $5.7(-10.48$ to 21.87$)$ \\
\hline Physical activity & $34(38$ to 44.19$)$ & $35.62(38.42$ to 44$)$ & $34(38.6$ to 41.15$)$ & $34(38.62$ to 42.9$)$ & 1. $(-0.2$ tol.1) \\
\hline
\end{tabular}

Data are means (SD) or median (percentile 25 to percentile 75) for non-normally distributed data. There were no significant differences between control and P.oleracea groups according to the independent t-test and Mann-Whitney test. ${ }^{a}$ Mean Difference (95\% Confidence Intervals). 
Table 3. Comparing of demographic data and anthropometric measurements in the control and P.oleracea groups at the baseline and end of 8 weeks.

\begin{tabular}{|c|c|c|c|c|c|}
\hline \multirow{2}{*}{ Parameter } & \multicolumn{2}{|c|}{ P.oleracea seeds group $(n=18)$} & \multicolumn{2}{|l|}{ Control group $(n=19)$} & \multirow{2}{*}{$\operatorname{MD}(95 \% C I)^{b}$} \\
\hline & Baseline(mean(SD)) & 8 weeks(mean(SD)) & Baseline(mean(SD)) & 8 weeks(mean(SD)) & \\
\hline Age(yr) & $44(808)$ & - & $46(9.6)$ & - & - \\
\hline Duration of asthma(yr) & $10(7.5)$ & - & $9.6(8.8)$ & - & - \\
\hline Height $(\mathrm{Cm})$ & $157.72(7.1)$ & - & $159.89(6.5)$ & - & - \\
\hline Weight(Kg) & $74.7(9.8)$ & $74.9(9.9)$ & $75.9(5.9)$ & $76.2(6.3)$ & $-0.19(-1.8$ to 1.4 \\
\hline BMI & $29.99(2.89)$ & $30.25(2.86)$ & $29.75(2.5)$ & $29.87(2.62)$ & $-0.07(-0.68$ to 0.54$)$ \\
\hline $\mathrm{WC}(\mathrm{Cm})$ & $100.8(8.01)$ & $102.1(8.43)$ & $100.3(7.96)$ & $99.7(16.8)$ & $2.17(-0.75$ to 5.9$)$ \\
\hline $\mathrm{HC}(\mathrm{Cm})$ & $104.63(5.58)$ & $106.6(5.06)^{\mathrm{a}}$ & $104.7(6.02)$ & $104.7(5.86)$ & $-2.13(-4.01 \text { to }-0.25)^{\mathrm{c}}$ \\
\hline WHR & $0.98(0.1)$ & $0.94(0.07)^{\mathrm{a}}$ & $0.95(0.09)$ & $0.95(0.1)$ & $0.04(-0.05 \text { to } 0.08)^{\mathrm{c}}$ \\
\hline
\end{tabular}

BMI, body mass index; WC, waist circumference; HC, hip circumference; WHR, waist-to-hip ratio; there were no significant differences between control and P.oleracea seeds groups according to the independent t-test. ${ }^{a} \mathrm{P}<0.05$, paired t-test. ${ }^{\mathrm{b}}$ Mean Difference (95\% Confidence Intervals). ${ }^{\mathrm{c}} \mathrm{P}<0.05$, analysis of covariance adjusted for baseline measurements.

At the baseline and end of this study, no significant differences in BMI, WC, HC and WHR were observed $(P>0.05)$. However, there were significant differences for the WC and WHR between two groups after adjusted for baseline measurements. Nevertheless, administration of P.oleracea caused to significant increase $(P<0.05)$ for $\mathrm{HC}$ in P.oleracea group compared with control. Furthermore, the waist-to-hip ratio in intervention group was significantly reduced with consumption of seeds after 8 weeks (Table3).

\section{Discussion}

Obesity is considered as a known risk factor for cardiovascular disease, diabetes and some cancers (1). Besides, epidemiological studies also exhibit the role of obesity in lung diseases like asthma (4) so that it is associated with increased severity of asthma in adults and children (21). According to these studies, modest weight reduction can effectively lead to improve asthma control, lung function and exacerbations $(22,23)$.

In this experiment aimed to study the effect of P.oleracea seeds on anthropometric measurements in asthmatic patients with overweight and obesity, no significant reduction was shown in weight and BMI during administration of seeds. In a study was done by Zakizadeh et al, receiving of $10 \mathrm{~g} \mathrm{P}$. oleracea seeds with $240 \mathrm{ml}$ low fat yoghurt for 5 weeks led to significant decrease of weight and BMI in diabetic patients (24). El-Sayed et al reported that consumption of $10 \mathrm{~g}$ P.oleracea seeds with $40 \mathrm{ml}$ skimmed yoghurt during 8 weeks was significantly reduced weight and BMI of diabetic patients (20). Samani et al found that 50-60g raw leaves and stems of this plant for 45 days can improve BMI in patients with $\mathrm{LDL}>100 \mathrm{ml} / \mathrm{dl}$ (15). In folk medicine of some countries like Yemen, P.oleracea was used to lose weight such that taking of one teaspoon of it before lunch and dinner with walking can lead to weight lost. These effects might stem from niacin and thiamine participation as coenzymes in metabolism of macronutrients (20). In Iranian traditional herbal medicines, P.oleracea was classified as a plant with cold and wet natures so it was considered to gain weight in people with warm and dry natures (25).

In present study, administration of seeds showed no significant difference for WC of asthmatic patients in intervention group but there were significant changes in the WC and WHR when adjusted for the baseline measurements. Thus, seeds resulted to reduction of WC and increase of WHR. Fat distribution is correlated with airway function. In fact, central obesity seriously influences mechanical functions of lungs (22). Waist circumference as an index for central obesity can anticipate the risk of asthma. According to some studies, central obesity develops an important role than obesity due to body mass index for determining late-onset asthma. In central obesity, fat mass increases its pressure to the diaphragm and decreases lung volumes and functions (4).

Asthmatic patients are prepared to gain weight and obesity because of limited physical activity and taking steroid drugs (26). At the end of eight weeks, our results exhibited no statistically significant changes about physical activity and daily intake of energy and macronutrients including carbohydrates, proteins and fats in both study groups. Therefore, we could control these parameters as likely confounders.

This study had some limitations. We could not find any placebo similar to P.oleracea seeds. Next, in spite of drugmatch, if the study was done without administration of any drugs, the more exact results was obtained because of probable drug interventions. While, it was impossible as a result of ethical issues and term of study ( 8 weeks).

Because of the importance of weight control in asthma and the possible effects of P.oleracea seeds on weight loss, it is recommended to repeat this study with more than $10 \mathrm{~g}$ /day and 8 weeks.

\section{Conclusion}

P.oleracea seeds were non-effective for reducing anthropometric measurements in overweight and obese patients with asthma. However, it is suggested that further studies are carried out to explore the effects of P.oleracea seeds for weight control in asthmatic patients. 


\section{Acknowledgment}

The authors would like to thank from all patients who participated in this study and respiratory lab staff of Imam Reza hospital. This article was written based on the data from a M.S thesis on nutrition and was financially supported by Vice chancellor for research, Tabriz University of Medical Sciences.

\section{References}

[1] Manion AB. Asthma and Obesity: The Dose Effect. Nursing Clinics of North America. 2013;48(1):151-8.

[2] Allan K, Devereux G. Diet and Asthma: Nutrition Implications from Prevention to Treatment. J Am Diet Assoc. 2011;111(2):258-68.

[3] Al-Afaleg NO, Al-Senaidy A, El-Ansary A. Oxidative stress and antioxidant status in Saudi asthmatic patients. Clinical Biochemistry. 2010;44(8-9).

[4] Song W-J, Kim S-H, Lim S, Park Y-J, Kim M-H, Lee S-M, et al. Association between obesity and asthma in the elderly population: potential roles of abdominal subcutaneous adiposity and sarcopenia. Annals of Allergy, Asthma \& Immunology. 2012.

[5] Dixon AE, Pratley RE, Forgione PM, Kaminsky DA, Whittaker-Leclair LA, Griffes LA, et al. Effects of obesity and bariatric surgery on airway hyperresponsiveness, asthma control, and inflammation. Allergy Clin. Immunol.. 2011;128(3):508-15.

[6] Mandal S, Hart N. Respiratory complications of obesity. Clinical Medicine. 2012;12(1):75-8.

[7] Cho YS, Moon H-B. The role of oxidative stress in the pathogenesis of asthma. Allergy Asthma Immunol. Res. 2010;2(3):183-7.

[8] Shah V, Shah V, Santani DD. Asthma and Plants used for Asthma-An Overview. Research Journal of Pharmacology and Pharmacodynamics. 2012;4(5):328-38.

[9] Al-Quraishy S, Dkhil MA, Abdel Moneim AE. Protective effects of Portulaca oleracea against rotenone mediated depletion of glutathione in the striatum of rats as an animal model of Parkinson's disease. Pesticide Biochemistry and Physiology. 2012;103(2):108-14.

[10] Liu L, Howe P, Zhou Y-F, Xu Z-Q, Hocart C, Zhang R. Fatty acids and B-carotene in Australian purslane (Portulaca oleracea) varieties. Journal of Chromatography A. 2000;893(1):207-13.

[11] Lim YY, Quah EPL. Antioxidant properties of different cultivars of Portulaca oleracea. Food Chemistry. 2007;103(3):734-40.
[12] Dkhil M, Abdel Moniem A, Al-Quraishy S, Saleh R. Antioxidant effect of purslane (Portulaca oleracea) and its mechanism of action. J Med Plant Research. 2011;5:1589-63.

[13] Mohamed-I Kotb E-S. Effects of Portulaca oleracea L. seeds in treatment of type-2 diabetes mellitus patients as adjunctive and alternative therapy. Journal of Ethnopharmacology.137(1):643-51.

[14] Hajzadeh MR, Rakhshandeh H, Esmaeilizadeh M, Ghorbani A Analgesic and anti-inflammatory effects of Portolaca oleracea extracts in mice \& rat. Koomesh. 2004;5(3,4):113-20.

[15] Gatreh-Samani K, Farrokhi E, Khalili B, Rafieian M, Moradi M. Purslane (Portulaca oleracea) effects on serum paraoxanase-1 activity. Journal of Shahrekord University of Medical Sciences. 2011;13(1):9-14. (persian)

[16] Zargar BA, Tabasum N, Masoodi MH, Ahmad B, Mir SR. Portulaca oleracea L. A Review. Journal of Pharmacy Research.4(9).

[17] Cui MZ, Liu H, Li CY. Changes of blood glucose in diabetic rats and the interventional effect of purslane. Chinese Journal of Clinical Rehabilitation. 2005;9(27):92-3.

[18] Movahedian A, Ghannadi A, Vashirnia M. Hypocholesterolemic effects of Purslane extract on serum lipids in rabbits fed with high cholesterol levels. Int J Pharmacol. 2007;3:285-9.

[19] Gong F, Li F, Zhang L, Li J, Zhang Z, Wang G. Hypoglycemic effects of crude polysaccharide from purslane. International journal of molecular sciences. 2009;10(3):880-8.

[20] El-Sayed M-IK. Effects of Portulaca oleracea L. seeds in treatment of type-2 diabetes mellitus patients as adjunctive and alternative therapy. Journal of ethnopharmacology.2011;137(1):643-51.

[21] Wood LG, Gibson PG. Adiponectin: The Link Between Obesity and Asthma in Women? American journal of respiratory and critical care medicine. 2012;186(1):1-2.

[22] Boulet LP. Asthma and obesity. Clinical \& Experimental Allergy. 2013;43(1):8-21.

[23] Ross KR, Hart MA. Assessing the relationship between obesity and asthma in adolescent patients: a review. Adolescent Health. 2013;4:39-49.

[24] Zaki E, Jazayeri S, Emany E, Gohari M, Esmaill M. Effect of Purslane seeds on glycemic parameters and serum lipid profiles in patients with type II diabetes: cross section clinical trial. Medical Research. 2013. (persian)

[25] shirazi aa, hadi smhm. makhzan aladvie. tehran: Tehran University of Medical Sciences - sabz Arang; 2011.(persian)

[26] Dixon A. The treatment of asthma in obesity. Expert Rev Respir Med 2012;6(3):331-40. 